\title{
Hybrid Multilevel Thresholding and Improved Harmony Search Algorithm for Segmentation
}

\author{
Erwin $^{1}$, Saparudin $^{2}$, Wulandari Saputri ${ }^{3}$ \\ ${ }^{1,3}$ Department of Computer Engineering, University of Sriwijaya, Indonesia \\ ${ }^{2}$ Department of Informatic Engineering, University of Sriwijaya, Indonesia
}

\begin{tabular}{l}
\hline Article Info \\
\hline Article history: \\
Received Apr 9, 2018 \\
Revised Jul 12, 2018 \\
Accepted Jul 22, 2018 \\
\hline
\end{tabular}

Keyword:

Image segmentation Improved harmony search Algorithm Multilevel thresholding

\begin{abstract}
This paper proposes a new method for image segmentation is hybrid multilevel thresholding and improved harmony search algorithm. Improved harmony search algorithm which is a method for finding vector solutions by increasing its accuracy. The proposed method looks for a random candidate solution, then its quality is evaluated through the Otsu objective function. Furthermore, the operator continues to evolve the solution candidate circuit until the optimal solution is found. The dataset used in this study is the retina dataset, tongue, lenna, baboon, and cameraman. The experimental results show that this method produces the high performance as seen from peak signal-to-noise ratio analysis (PNSR). The PNSR result for retinal image averaged $40.342 \mathrm{~dB}$ while for the average tongue image $35.340 \mathrm{~dB}$. For lenna, baboon and cameramen produce an average of $33.781 \mathrm{~dB}, 33.499 \mathrm{~dB}$, and $34.869 \mathrm{~dB}$. Furthermore, the process of object recognition and identification is expected to use this method to produce a high degree of accuracy.
\end{abstract}

Copyright $@ 2018$ Institute of Advanced Engineering and Science. All rights reserved.

\section{Corresponding Author:}

Erwin,

Department of Computer Engineering,

University of Sriwijaya,

Jl. Raya Palembang-Prabumulih KM.32 Indralaya Ogan Ilir 30662, Palembang, Indonesia.

Email: erwin@unsri.ac.id

\section{INTRODUCTION}

Segmentation is a process in image processing that processes the original image into constituent or object areas. The purpose of segmentation is to separate an object from the whole image. Currently, image processing can be applied very widely in various fields, for example in the fields of astronomy, archeology, and even biomedical. Image processing on biomedicine has been widely used, including face detection [1], iris [2], ear, and tongue. Using image processing technique such as level set and region growing, an ophthalmologist may know the disease through eye retina and the technology can know the disease in the eye retina [3]. One of research conducted by [4] aims to classify types of diseases through the color of the tongue with a success rate of $91.99 \%$ accuracy.

Another research on tongue image segmentation was done by [5] with 70\% accuracy and [6] using active contour model method which gives $75 \%$ of accuracy. Additionally, [7] combines region-based and edge-based methods in segmenting images. Detection and classification of the retinal changes for Diabetic Retinopathy monitoring were performed by [8]. This research extract retrospective changes in longitudinal crack and tyrosinetopathy showing $97 \%$ detection rate and $99.3 \%$ classification rate. Reseach from [9] has been conducted to automatic segmentation and identification of diabetics through retinal vessels.

The method used for segmentation is Gabor wavelet transformation. Therefore, the results obtained that traditional features do not detect early proliferative retinopathy. For the classification method used is the wavelet method that is able to group the retinal blood vessels in accordance with the presence or absence of 
proliferative retinopathy. The success rate of this method decreased to $50 \%$ for the identification from the previous stages. Based on the description above it is necessary to optimize segmentation process, in order to improve the quality of segmentation results.

The threshold method is one of the most widely used methods of image segmention [10],[11]. Segmentation process for two segmen of image is called bilevel thresholding and more than two segmen is called multilevel thresholding. Otsu and Kapur are two classical methods of multilevel thresholding. Segmentation using multilevel thresholding requires a long computation time and involves a large calculation. In order to solve this problem, optimization methods should be applied [12]. Several optimization methods that have been successfully applied in multilevel thresholding, including Genetic Algorithms (GA) and Particle Swarm Optimization (PSO). One interesting example of multilevel thresholding with GA is shown in [13], [14]. Furthermore, [15] using multilevel thresholding and Improved Differential Search Algorithm (IDSA) to the segment. Image segmentation with PSO-based multilevel thresholding was done by [16]-[18].

Harmony search algorithm (HSA) is an optimization algorithm inspired by the improvisation process of jazz musicians, from the phenomenon of opera music that consists of various musical instruments and produces beautiful melodies. The algorithm was introduced by Geem et al. [19] [20]. In previous research, multilevel thresholding with HSA was used in segmenting the image with two methods of thresholding, ie Otsu, and Kapur. Multilevel thresholding with Otsu that has been optimized using HSA shows better results compared to Kapur [19]. Improved harmony search algorithm (IHSA) has also been applied in segmentation problems for brain images [21]. The study uses IHSA and combines it with fuzzy clustering algorithms. However, there remains a weakness in the study is shown with the PSNR value that is not high enough.

Research on retinal image segmentation has been done by [22], [23] aiming to simplify or change image representation into something that is easier to analyze. [24] who have conducted a retinal image study by proposing a computerized technique for extracting retinal vessels. In addition, [25] and [26] conducted a retinal image study for classification segmentation of retinal disease types using different methods. [27] segmenting the retinal vessels using a single oriented mask filter.

The experimental results show that the proposed method outperforms a single oriented mask filter [28]. Segmenting the area around the retina by using adaptive superpixalation that is used to detect the disease around the retina area. Experimental evaluation gives better results with 96\% accuracy [29]. Conducted a study to identify the early diagnosis of epileptic diseases of glaucoma, diabetic retinopathy, macular degeneration, hypertensive retinopathy, and arteriosclerosis. There are two methods of doing this segmentation by using the method of extraction of blood vessel centerline pixels and iterative region growing.

This reseach proposed a novel method to improve segmentation performance using multilevel thresholding with HSA and IHSA. This paper introduces a new method of hybrid multilevel thresholding and improved harmony search algorithm (MT-IHSA). The parameters used differ from the HSA that lies in the adjustment of pitch adjusting rate (PAR) and bandwidth (BW) [21]. Where this method combines IHSA method and thresholding using Otsu. This research is expected to show more optimal results of PSNR than previous research.

\section{OTSU MULTILEVEL THRESHOLDING FOR IMAGE SEGMENTATION}

Image segmentation is a process to separate the image to the foreground and background so it is easier to analyze [30]. The process of image segmentation is very important, the higher the accuracy level generated at the segmentation stage the better the object recognition process [31]. Thresholding is known as a non-linear operation that is important in image segmentation [32]. The basic idea of thresholding is to choose an optimal gray-level threshold value to separate objects and backgrounds based on gray-level distribution [33].

There are two types of thresholding, ie global and local. Otsu is a global thresholding introduced by Otsu in 1979 [34]. This method is widely used because it includes a simple and effective method. Otsu uses the maximum variance value of class differences as the image segmenting criteria. By taking the intensity level (L) of the grayscale or RGB image, the probability distribution of the intensity value of the image can be calculated as follows Equation 1 [19]:

$$
P h_{i}^{c}=\frac{h_{i}^{c}}{N P}, \sum_{i=1}^{N P} P h_{i}^{c}=1,
$$

where; $\mathrm{i}=$ intensity level $(0 \leq \mathrm{i} \leq \mathrm{L}-1)$ 
$\mathrm{c}=$ component of the image, depending on grayscale or RGB

$\mathrm{NP}=$ the number of pixels in the image

$h_{i}^{c}=$ histogram (the number of pixels corresponding to the intensity level in c)

$P h_{i}^{c}=$ probability of distribution

The simplest bilevel segmentation can be defined as follows Equation 2:

$$
C_{1}=\frac{P h_{1}^{c}}{\omega_{0(t h)}^{c}}, \ldots \frac{P h_{t h}^{c}}{\omega_{0(t h)}^{c}}, C_{2}=\frac{P h_{t h+1}^{c}}{\omega_{1(t h)}^{c}}, \ldots \frac{P h_{L}^{c}}{\omega_{1(t h)}^{c}}
$$

Where $\omega_{o}(t h)$ and $\omega_{1}(t h)$ is the distribution probability for $C_{1}$ and $C_{2}$ where :

$$
\begin{aligned}
& \omega_{0}^{c}(t h)=\sum_{i=1}^{t h} P h_{i}^{c}, \omega_{1}^{c}(t h)=\sum_{i=t h+1}^{L} P h_{i}^{c} \\
& \mu_{0}^{c}=\sum_{i=1}^{t h} \frac{i P h_{i}^{c}}{\omega_{0}^{c}(t h)}, \mu_{1}^{c}=\sum_{i=t h+1}^{L} \frac{i P h_{i}^{c}}{\omega_{1}^{c}(t h)}
\end{aligned}
$$

Otsu Variance between classes can be calculated by following Equations 3 and 4 as follows:

$$
\begin{aligned}
& {\sigma^{2}}^{c}=\sigma_{1}^{c}+\sigma_{2}^{c}, \\
& \sigma_{1}^{c}=\omega_{0}^{c}\left(\mu_{0}^{c}+\mu_{T}^{c}\right)^{2}, \sigma_{2}^{c}=\omega_{1}^{c}\left(\mu_{1}^{c}+\mu_{1}^{c}\right)^{2}
\end{aligned}
$$

Where $\mu_{T}^{c}=\omega_{0}^{c} \mu_{o}^{c}+\omega_{1}^{c} \mu_{1}^{c}$ and $\omega_{0}^{c}+\omega_{1}^{c}=1$.

The following is an objective function based on the value of $\sigma_{1}^{c}$ and $\sigma_{2}^{c}$ :

$$
J(\boldsymbol{T H})=\max \left(\sigma^{2^{c}}(\boldsymbol{T H})\right)
$$

With $0 \leq t h_{i} \leq L-1, i=1,2, \ldots, k$, where $t h=t h_{1}, t h_{2}, \ldots, t h_{k-1}$ is a vector containing several thresholds and then the variance is computed as Equation 6 as:

$$
\sigma^{2^{c}}=\sum_{i=1}^{k} \sigma_{i}^{c}
$$

\section{IMPROVED HARMONY SEARCH ALGORITHM}

HSA is a new metaheuristic optimizer introduced by Zong Woo Geem, Joong Hoon Kim, and G.V. Loganathan in 2001, this method yielded very good results in the field of optimization [32]. HSA is inspired by improvised jazz musicians, from the phenomenon of opera music and produces beautiful melodies. The advantages of HSA compared to other optimization techniques are: HSA is a metaheuristic algorithm and does not require configuration values based on determinant variables, HSA uses stochastic random searches, HSA does not require derivative information, has several parameters, and can be easily adopted in a wide range of optimization problems. The steps in the HSA process are as follows [21]:

a. Minimalize $f(x)$ subject to $x_{i} \in X_{i}=1,2, \ldots, \mathrm{N}$

where; $f(x)=$ objective function

$x=$ collection of decision variables $X_{i}$

$\mathrm{N}=$ total of decision variables

$X_{i}=$ collection of probability range for each decision variables

In this step, the HSA parameters are specified. HSA parameters consist of the number of solution vectors in harmony memory (HM) called harmony memory size (HMS), harmony memory consideration rate (HMCR), pitch adjusting rate (PAR), and termination criteria called a number of improvisations (NI). Here are the parameters in the HSA [33] :

HMS: total vectors simultaneously in Harmony Memory (HM). Values vary from 1 to 1000.

HMCR: the level or percentage of HSA values taken from HM, the value varies from 0.7 to 0.99

PAR: pointer at the level or percentage of the close value, the value varies from $0.1-0.5$

Number of NI: indicates the iteration number in the optimization algorithm.

b. Harmony Memory (HM) Initialization Equation 7.

At this stage, the HM matrix is filled with HMS which is the solution vector randomized by 


$$
H M=\left[\begin{array}{ccccc}
x_{1}^{1} & x_{2}^{1} & \cdots & x_{N-1}^{1} & x_{N}^{1} \\
x_{1}^{2} & x_{2}^{2} & \cdots & x_{N-1}^{2} & x_{N}^{2} \\
\vdots & \vdots & \cdots & \vdots & \vdots \\
x_{1}^{H M S-1} & x_{2}^{H M S-1} & \cdots & x_{N-1}^{H M S-1} & x_{N}^{H M S-1} \\
x_{1}^{H M S} & x_{2}^{H M S} & \cdots & x_{N-1}^{H M S} & x_{N}^{H M S}
\end{array}\right]
$$

c. Improvisation of New Harmony

Improvisation is processed to obtain new harmony. The new vector harmonies can be obtained under the following rules:

a) Choose one of the values of HSA Harmony (HMCR)

b) Select one value closest to HSA memory (tone adjustment)

c) Select a random value from a range of possible values (randomization)

In memory considerations, first determine the value of the variable $\left(x_{1}^{1}\right)$ for a new vector taken from one of the values in the predefined HM range $\left(x_{1}^{\prime 1}-x_{1}^{\prime H M S}\right)$. The value of the other determinant variables is picked in the same way. HMCR (0 to 1) is the step of selecting a random value from a possible range value. At this stage, HM considerations, tone adjustments, or random selection are applied alternately for each new harmony vector variable.

d. Update HM

In this step, if the new harmony vector is better than the existing harmony in HM rather than based on the value of the objective function, the new harmony can enter HM, and the worst harmony will not be included in HM.

e. Check for termination criteria

If the termination criteria are met (maximum NI) of the computation process will be stopped. If not, repeat steps 3 and 4. The main difference between HSA and IHSA is on the PAR and BW adjustment path. IHSA improves the performance of HSA algorithms and eliminates weak points. This method uses PAR and BW in step 3 (improvisation). Pseudocode for original IHSA algorithm:

Step 1. Initialize parameters HMS, HMCR, $c, P A R_{\max }, B W_{\text {min }}, B W_{\text {max }}$, and NI.

Step 2. Initialize HM and calculate $\mathrm{f}(\mathrm{x})$ of each harmony vector.

Step 3. Improvise new harmony.

for iteration $\leq$ number of variable

$\mathrm{PAR}=P A R_{\text {min }}+\frac{\left(P A R_{\max }-P A R_{\min }\right)}{N I} \times \mathrm{gn}$

$\mathrm{c}=\mathrm{in}\left(B W_{\min } / B W_{\max }\right) / \mathrm{NI}$

$\mathrm{BW}=B W_{\text {max }} \mathrm{x} \exp (\mathrm{c} \times \mathrm{gn})$

for(all variable)

if $\operatorname{rand}() \leq \mathrm{HMCR}$

$x_{i}^{\prime}=x_{i}^{j}(\mathrm{j}=1,2, \ldots, \mathrm{HMS})($ choose value from $\mathrm{HM})$

if $\operatorname{rand}() \leq$ PAR

$$
x_{i}^{\prime}=x_{i}^{\prime} \pm \operatorname{rand}() \times \mathrm{BW}
$$

endif

else

(choose a random value of variable)

$x_{i}^{\prime}=P V B_{\text {lower }}+\operatorname{rand}() \times\left(P V B_{\text {upper }}-P V B_{\text {lower }}\right)$ endif

endfor

endfor

Step 4.Update HM.

if(new solution $\leq$ worst solution)

replace the worst harmony in HM with the new harmony

endif

Step 5.Check stopping criteria. If NI is completed, terminate computation; otherwise go back to Step 3.

\section{PROPOSED METHOD: HYBRID MULTILEVEL THRESHOLDING AND IMPROVED} HARMONY SEARCH ALGORITHM (MT-IHSA)

This method is a hybrid between two stratified thresholding methods by Otsu and IHSA, which is MT-IHSA. The proposed method is to search randomly in the histogram as a candidate, then evaluate its 
quality by using the objective function by Otsu. Furthermore, IHSA operators will evolve on candidate strings until the most optimal solution is found. Pseudocode for MT-IHSA algorithm :

Step 1. Obtain histograms.

Step 2. Calculate the probability distribution.

Step 3. Initialize the IHSA parameters: HMS, HMCR, $c, P A R_{\max }, B W_{\min }, B W_{\max }$, and NI.

Step 4. Initialize a Harmony Memory (HM).

Step 5 Calculate Otsu variance.

Step 6. Evaluate objective function

Step 7. Improvise a new harmony.

Step 8 Update the HM

Step 9. If NI is completed or the stop criteria is satisfied, then jump to Step 10, otherwise go back to Step 6 .

Step 10.Select the harmony that has the best objective function value.

Step 11 Apply the best thresholds values to the image.

\section{RESULT AND DISCUSSION}

The dataset used in this experiment consisted of two datasets, the retinal dataset obtained from STructured Analysis of the Retina (Stare) with 450 retina images and tongue dataset obtained from biometric research center (BRC) with $12 \mathrm{jpg}$ format the image of the tongue. In addition, lenna, baboon, and cameraman images are also used for testing the proposed method. The parameters and values used in the MTIHSA presented in Table 1 consist of NI, HMS, HMCR, PAR Min, PAR Max, BW Min, and BW Max. The value of the parameters using [19], namely:

Table 1. Parameters used in MT-IHSA

\begin{tabular}{cc}
\hline Parameters & Values \\
\hline NI & 2,000 \\
HMS & 5 \\
HMCR & 0.9 \\
PAR Min & 0.01 \\
PAR Max & 0.99 \\
BW Min & 0.001 \\
BW Max & 0.1 \\
\hline
\end{tabular}

Table 2 is the result of image segmentation using MT-IHSA with threshold value 5, a histogram of 5 types of the image shows a very significant difference. In the original image, the resulting histogram still has red, green, blue (RGB). For the grayscale image, the resulting histogram has a gray color, but the resulting color still has a very high color so it is still difficult to distinguish between foreground and background. The third histogram is the resulting histogram for image implementation of the MT-IHSA. The colors produced after going through the MT-IHSA process have very few color components. Because the colors with applied MT-IHSA colors applied are more likely to binary.

Table 2 shows the results of the application of segmentation using multilevel thresholding. Before applying the MT-IHSA first the histogram value of the original image is taken in order to see the color pixel values contained in the original image. Then do the grayscale process to reduce the pixels contained in RGB color. The histogram obtained from the grayscale has fewer pixel values. Then the application of segmentation using MT-IHSA. The pixels obtained are very low. Background and foreground are completely separated. Although the resulting pixels are very low, the resulting image quality is very good and is seen more clearly using MT-IHSA.

The threshold value specified in this testing process is th=2,3,4,5. PSNR is the value of comparison between the maximum pixel value of the image using the mean square error (MSE). MSE is the average error value between the segmented image and the original image. The greater the PSNR results show better image quality. PSNR is expressed in decibels $(\mathrm{dB})$. The value of PSNR can be categorized well if $>=30 \mathrm{~dB}$, it can be formulated as follows with Equation 8 and root mean square error (RMSE) value with Equation 9:

$$
\begin{aligned}
& P S N R=20 \log _{10}\left(\frac{255}{R M S E}\right) \\
& R M S E=\sqrt{\frac{\sum_{i=1}^{r o} \sum_{j=1}^{c o}\left(I_{0}^{c}(i, j)-I_{t h}^{c}(i, j)\right)}{\operatorname{row} x \operatorname{col}}}
\end{aligned}
$$


where; $I_{0}^{c}=$ original image

$I_{t h}^{c}=$ segmented image

row $\mathrm{x} \mathrm{col=Total}$ amount of image rows and columns

Another goal of using PSNR is to evaluate the similarity between the segmented image and the original image. Comparison of PNSR results using retinal image and tongue image in Table 3. PNSR on a retinal image is greater than with PNSR of tongue image.

In Table 3, the results of the application of segmentation on retinal image and tongue have been obtained. PSNR generated above $30 \mathrm{~dB}$. That is, the result of segmentation is done successfully because it has exceeded $30 \mathrm{~dB}$. The bigger the PNSR gets, the better the pixel gets. Segmentation performed on the tongue image receives a lower PSNR value than that of the retinal image.

Table 2. Results of Image Segmentation and Histogram with MT-IHSA with Threshold Value 5

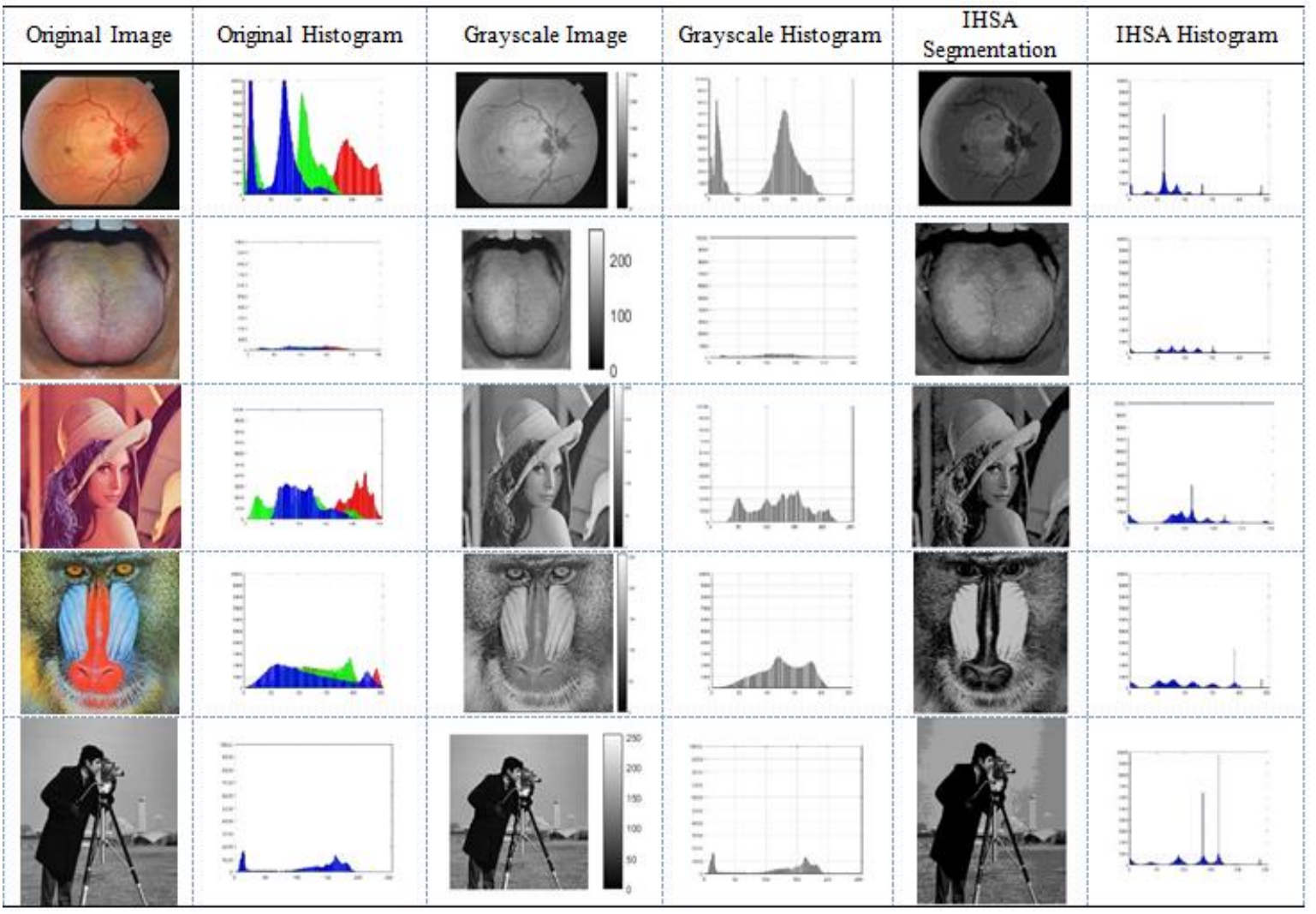

Table 3. Comparison of PSNR Value and Segmentation Threshold Results with MT-IHSA Using Retina Image and Tongue Image

\begin{tabular}{|c|c|c|c|c|c|c|}
\hline \multirow{2}{*}{ Image } & \multirow{2}{*}{ th } & \multicolumn{2}{|c|}{ PNSR } & \multirow{2}{*}{ image } & \multicolumn{2}{|c|}{ PNSR } \\
\hline & & Retina Image & Tongue Image & & Retina Image & Tongue Image \\
\hline \multirow{4}{*}{1} & 2 & 43.36 & 35.558 & \multirow{4}{*}{7} & 35.578 & 34.88 \\
\hline & 3 & 45.121 & 36.074 & & 39.68 & 35.617 \\
\hline & 4 & 45.121 & 37.198 & & 40.349 & 36.061 \\
\hline & 5 & 48.131 & 38.015 & & 41.141 & 37.173 \\
\hline \multirow{4}{*}{2} & 2 & 41.141 & 33.745 & \multirow{4}{*}{8} & 39.68 & 31.992 \\
\hline & 3 & 42.11 & 34.761 & & 42.11 & 34.857 \\
\hline & 4 & 43.36 & 35.482 & & 45.121 & 36.426 \\
\hline & 5 & 45.121 & 36.013 & & 48.131 & 36.426 \\
\hline \multirow{4}{*}{3} & 2 & 40.349 & 34.674 & \multirow{4}{*}{9} & 38.588 & 32.037 \\
\hline & 3 & 39.68 & 35.468 & & 39.68 & 34.279 \\
\hline & 4 & 42.11 & 36.035 & & 40.349 & 35.288 \\
\hline & 5 & 40.349 & 37.138 & & 39.68 & 35.936 \\
\hline \multirow{4}{*}{4} & 2 & 41.141 & 34.76 & \multirow{4}{*}{10} & 39.09 & 31.595 \\
\hline & 3 & 42.11 & 35.657 & & 37.339 & 32.908 \\
\hline & 4 & 43.36 & 36.377 & & 38.131 & 35.306 \\
\hline & 5 & 45.121 & 37.307 & & 39.68 & 35.926 \\
\hline
\end{tabular}


Table 3. Comparison of PSNR Value and Segmentation Threshold Results with MT-IHSA Using Retina Image and Tongue Image

\begin{tabular}{|c|c|c|c|c|c|c|}
\hline \multirow{2}{*}{ Image } & \multirow{2}{*}{ th } & \multicolumn{2}{|c|}{ PNSR } & \multirow{2}{*}{ image } & \multicolumn{2}{|c|}{ PNSR } \\
\hline & & Retina Image & Tongue Image & & Retina Image & Tongue Image \\
\hline \multirow{4}{*}{5} & 2 & 33.079 & 35.109 & \multirow{4}{*}{11} & 38.131 & 33.115 \\
\hline & 3 & 34.514 & 36.012 & & 38.131 & 34.594 \\
\hline & 4 & 36.991 & 36.806 & & 39.68 & 35.653 \\
\hline & 5 & 37.339 & 37.635 & & 41.141 & 36.082 \\
\hline \multirow{4}{*}{6} & 2 & 39.1 & 33.673 & \multirow{4}{*}{12} & 33.981 & 34.419 \\
\hline & 3 & 40.349 & 33.822 & & 34.707 & 35.032 \\
\hline & 4 & 41.141 & 34.814 & & 36.67 & 36.032 \\
\hline & 5 & 42.11 & 35.56 & & 37.339 & 36.981 \\
\hline
\end{tabular}

Segmentation from [28] is using single oriented mask filter, the result of the segmentation has increased but the resulting weight has not been maximized. The disadvantage of this method is when processing the results, the time is done long enough so that the process is slower. As for the process of MTIHSA, process data is processed faster and the results obtained exceeds $30 \mathrm{~dB}$. The next method used for segmentation is by using the Gabor wavelet transformation. But for the results obtained that traditional features do not detect early proliferative retinopathy. Percentage of success is only $50 \%$. Of all the methods described, we can see the comparison that MT-IHSA is an excellent method for segmentation process.

Figure 1 is the result of the segmentation comparison by using the multi thresholding harmony search algorithm (MT-HSA) performed by [19] with the MT-IHSA proposed method using lenna, baboon, and cameramen images. Results from MT-HSA, PNSR values obtained below $30 \mathrm{~dB}$. The pixel values obtained do not match the default value of $30 \mathrm{~dB}$. This means that the implementation using MT-HSA segmentation is not appropriate and is still below the average. However, for segmentation results using MTIHSA, the segmentation obtained exceeds the $30 \mathrm{~dB}$ limit. Segmentation with the application of MT-IHSA shows good quality and success because the pixels produced are excellent and also exceed $30 \mathrm{~dB}$.

To compare it using other methods performed by [35] using the method of Tongue Color, Texture, and Geometry Features(CTGF), [19] with Multilevel Thresholding harmony search algorithm (MT-HSA) method, and [36] with Multilevel Thresholding Firefly Algorithm method (MT-FA) and Multilevel Thresholding Social Spider Algorithm (MT-SSA) are presented as Figure 2.

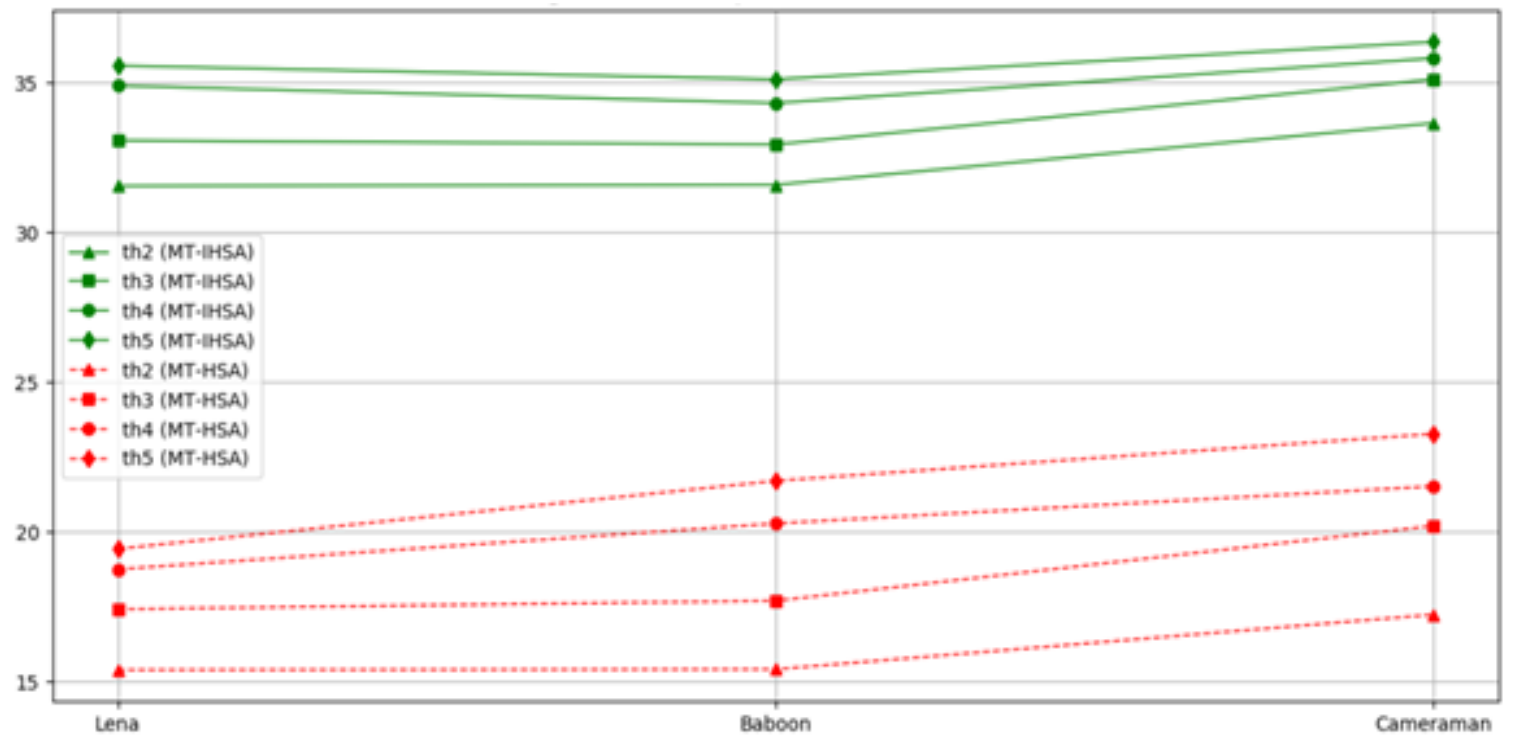

Figure 1. Diagram PSNR comparison MT-HSA and MT-IHSA

So the comparison of PNSR results between the proposed method (MT-IHSA) with MT-HSA, Color, Texture, and Geometry (CTGF), MT-FA and MT-SSA methods resulted in the highest PNSR score. Increasing the value of PNSR shows that the results of image segmentation with the proposed method produce the best segmentation quality compared with other methods. 


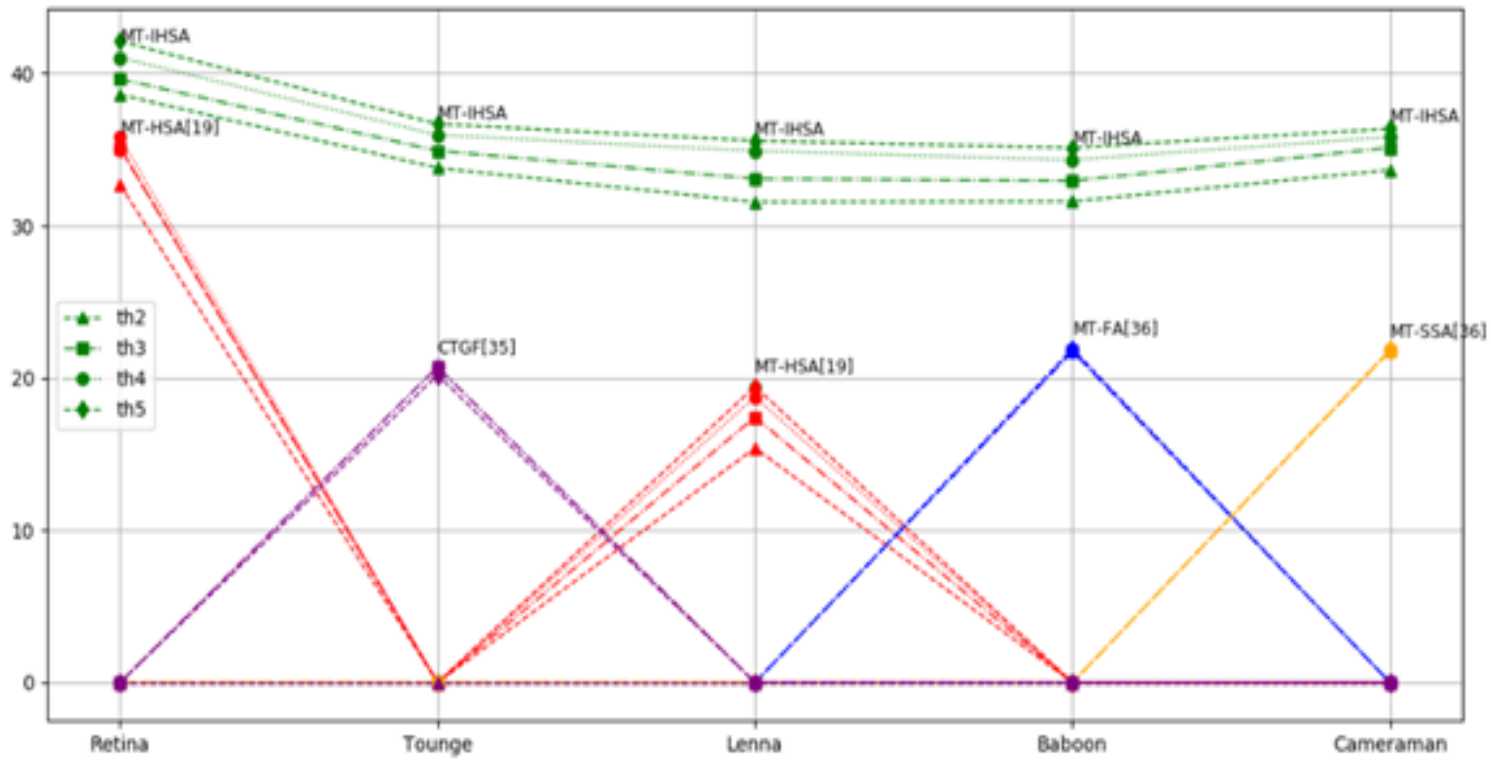

Figure 2. Diagram PSNR comparison of comparison of the PNSR value of segmentation method of MTIHSA, MT-HSA, CTGF, MT-FA, MT-SSA with MT-IHSA

\section{CONCLUSION}

In this study, we propose a new method of multilevel threshold hybrid with improved harmony search algorithm. This method is a hybrid between the IHSA algorithm and the objective function of multilevel thresholding using the Otsu method. The indicator used in this study to evaluate the performance of MT-IHSA is PSNR. The results of MT-IHSA experiments implemented on the retina image are higher than the image of the tongue image, but for the results obtained, the image of the tongue produces excellent segmentation compared to the retinal image. Likewise, for the image of Lena, baboon, and cameraman, PNSR produced after applying MT-IHSA increased. Previously for the image of Lenna, baboon, and cameramen applied using MT-HSA with PNSR result below 30dB. The comparison of segmentation using other method yields the PNSR value using the highest MT-IHSA. The level of illumination of an object is very influential for segmentation so that the results obtained more clearly. For the tongue image results showed better PSNR results than previous studies higher than $30 \mathrm{~dB}$.

The PNSR result for retinal image averaged $40,342 \mathrm{~dB}$ while for the average tongue image 35.340 dB. For lenna, baboon and cameraman produce average PNSR $33.781 \mathrm{~dB}, 33.499 \mathrm{~dB}$ and $34.869 \mathrm{~dB}$ respectively. Furthermore, the process of object recognition and identification is expected to use this method to produce a high degree of accuracy

\section{ACKNOWLEDGEMENTS}

This paper is partly supported by Direktorat Riset dan Pengabdian Masyarakat, Direktorat Jenderal Penguatan Riset dan Pengembangan, Kementerian Riset, Teknologi dan Pendidikan Tinggi Indonesia and Rector of University of Sriwijaya.

\section{REFERENCES}

[1] M. Fachrurrozi, Erwin, Saparudin, and Mardiana, "Multi-object face recognition using Content Based Image Retrieval (CBIR)," in 2017 International Conference on Electrical Engineering and Computer Science (ICECOS), 2017, pp. 193-197.

[2] M. Fachrurrozi and M. Mujtahid, "Iris Image Recognition Based on Independent Component Analysis and Support Vector Machine,” Telkomnika, vol. 13, no. 2, pp. 597-603, 2015.

[3] Y. Qian Zhao, X. Hong Wang, X. Fang Wang, and F. Y. Shih, "Retinal vessels segmentation based on level set and region growing," Pattern Recognit., vol. 47, no. 7, pp. 2437-2446, 2014.

[4] D. Zhang, H. Zhang, and B. Zhang, "Tongue Color Analysis for Medical Application,” Tongue Image Anal., vol. 2013, pp. 207-223, 2017.

[5] H. Murat et al., "Feature Extraction and Analysis on $\{\mathrm{CT}\}$ Image of Xinjiang Local Liver Hydatid by Using Gray- 
scale Histograms,” Sci. \&amp; Technol. Rev., vol. 30, no. 6, pp. 66-70, 2010.

[6] Saparudin, Erwin, and M. Fachrurrozi, “Tongue Segmentation Using Active Contour Model," in IAES International Conference on Electrical Engineering, Computer Science and Informatics IOP Publishing, 2017, pp. 1-6.

[7] K. Wu and D. Zhang, "Robust tongue segmentation by fusing region-based and edge-based approaches," Expert Syst. Appl., vol. 42, no. 21, pp. 8027-8038, 2015.

[8] H. Narasimha-Iyer et al., "Robust Detection and Classification of Longitudinal Changes in Color Retinal Fundus Images for Monitoring Diabetic Retinopathy," IEEE Trans. Biomed. Eng., vol. 53, no. 6, pp. 1084-1098, 2006.

[9] H. F. Jelinek, M. J. Cree, J. J. G. Leandro, J. V. B. Soares, R. M. Cesar, and a Luckie, "Automated segmentation of retinal blood vessels and identification of proliferative diabetic retinopathy.," J. Opt. Soc. Am. A. Opt. Image Sci. Vis., vol. 24, no. 5, pp. 1448-1456, 2007.

[10] D. Zhou and H. Zhou, "Minimisation of local within-class variance for image segmentation," IET Image Process., vol. 10, no. 2, pp. 608-615, 2016.

[11] X. Zheng, H. Ye, and Y. Tang, "Image bi-level thresholding based on gray level-local variance histogram," Entropy, vol. 19, no. 5, pp. 1-8, 2017.

[12] K. Chen, Y. Zhou, Z. Zhang, M. Dai, Y. Chao, and J. Shi, "Multilevel Image Segmentation Based on an Improved Firefly Algorithm," Math. Probl. Eng., vol. 2016, pp. 1-12, 2016.

[13] S. Manikandan, K. Ramar, M. Willjuice Iruthayarajan, and K. G. Srinivasagan, "Multilevel thresholding for segmentation of medical brain images using real coded genetic algorithm," Meas. J. Int. Meas. Confed., vol. 47, no. 1, pp. 558-568, 2014.

[14] S. Kotte, P. Rajesh Kumar, and S. K. Injeti, “An efficient approach for optimal multilevel thresholding selection for gray scale images based on improved differential search algorithm," Ain Shams Eng. J., 2015.

[15] M. H. Mozaffari and W.-S. Lee, "Convergent heterogeneous particle swarm optimisation algorithm for multilevel image thresholding segmentation," IET Image Process., vol. 11, no. 8, pp. 605-619, 2017.

[16] D. Mishra, I. Bose, U. Chandra De, and B. Pradhan, "A multilevel image thresholding using particle swarm optimization," Int. J. Eng. Technol., vol. 6, no. 2, pp. 1204-1211, 2014.

[17] Y. Liu, C. Mu, W. Kou, and J. Liu, "Modified particle swarm optimization-based multilevel thresholding for image segmentation," Soft Comput., vol. 19, no. 5, pp. 1311-1327, 2015.

[18] H. Maryam, A. Mustapha, and J. Younes, "A multilevel thresholding method for image segmentation based on multiobjective particle swarm optimization," IEEE, pp. 0-5, 2017.

[19] D. Oliva, E. Cuevas, G. Pajares, D. Zaldivar, and M. Perez-cisneros, "Multilevel Thresholding Segmentation Based on Harmony Search Optimization," J. Appl. Math., vol. 2013, 2013.

[20] Z. W. Geem, J. H. Kim, and G. Loganathan, "A New Heuristic Optimization Algorithm: Harmony Search," Simulation, vol. 76, no. 2, pp. 60-68, 2001.

[21] M. Mahdavi, M. Fesanghary, and E. Damangir, "An improved harmony search algorithm for solving optimization problems," Appl. Math. Comput., vol. 188, no. 2, pp. 1567-1579, 2007.

[22] B. Shuai, Z. Zuo, B. Wang, G. Wang, and S. Member, "Scene Segmentation with DAG-Recurrent Neural Networks," vol. 8828, no. c, 2017.

[23] Z. Yang, Y. Shufan, G. Li, and D. Weifeng, "Segmentation of MRI Brain Images with an Improved Harmony Searching Algorithm," Biomed Res. Int., vol. 2016, 2016.

[24] J. Dash and N. Bhoi, "A Thresholding Based Technique to Extract Retinal Blood Vessels from Fundus Images," Futur. Comput. Informatics J., 2017.

[25] C. Zhu et al., "Retinal vessel segmentation in colour fundus images using Extreme Learning Machine," Comput. Med. Imaging Graph., vol. 55, pp. 68-77, 2017.

[26] J. H. Tan, U. R. Acharya, S. V. Bhandary, K. C. Chua, and S. Sivaprasad, "Segmentation of optic disc, fovea and retinal vasculature using a single convolutional neural network," J. Comput. Sci., vol. 20, pp. 70-79, 2017.

[27] F. Kasmin, A. Abdullah, and A. S. Prabuwono, "Retinal Blood Vessel Segmentation Using Ensemble of Single Oriented Mask Filters," IJECE Int. J. Electr. Comput. Eng., vol. 7, no. 3, p. 1414, 2017.

[28] N. Singh and R. Gill, "Retinal Area Segmentation using Adaptive Superpixalation and its Classification using RBFN," IJECE Int. J. Electr. Comput. Eng., vol. 6, no. 6, p. 2674, 2016.

[29] R. Akhavan and K. Faez, "Two novel retinal blood vessel segmentation algorithms," IJECE Int. J. Electr. Comput. Eng., vol. 4, no. 3, pp. 398-410, 2014.

[30] R. Dass and S. Devi, "Image Segmentation Techniques," IJECT, vol. 3, no. 1, pp. 66-70, 2012

[31] R. C. Gonzales and R. E. Wood, Digital Image Processing. Prentice Hall, 2005.

[32] D. Manjarres et al., "A survey on applications of the harmony search algorithm," Eng. Appl. Artif. Intell., vol. 26, no. 8 , pp. $1818-1831,2013$.

[33] M.-A. Al-Betar and A.-T. Khader, "A harmony search algorithm for university course timetabling," Ann. Oper. Res., vol. 194, no. 1, pp. 3-31, 2012.

[34] M. H. J. Vala and A. Baxi, "A review on Otsu image segmentation algorithm," Int. J. Adv. Res. Comput. Eng. Technol., vol. 2, no. 2, pp. 387-389, 2013.

[35] B. Zhang, B. V. K. V. Kumar, and D. Zhang, "Detecting Diabetes Mellitus and Non-Proliferative Diabetic Retinopathy using Tongue Color, Texture , and Geometry Features," no. c, 2013.

[36] R. Singh, P. Agarwal, M. Kashyap, and M. Bhattacharya, "Kapur' s And Otsu's Based Optimal Multilevel Image Thresholding Using Social Spider and Firefly Algorithm," no. v, pp. 2220-2224, 2016. 


\section{BIOGRAPHIES OF AUTHORS}
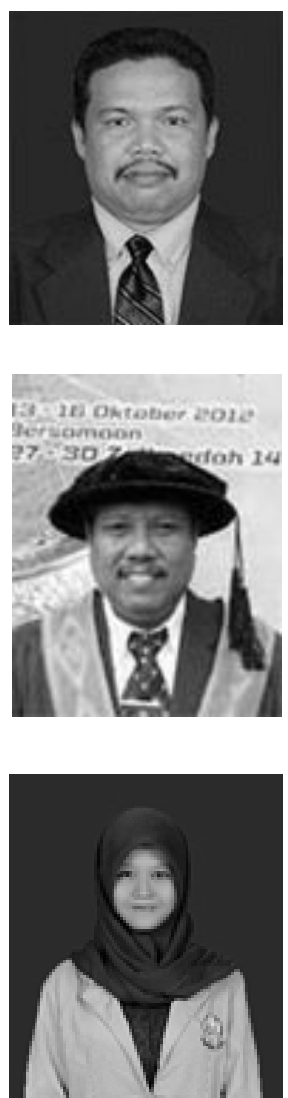

Erwin was born in Palembang, Indonesian, in 1971. He received the Bachelor degree in Mathematics from the University of Sriwijaya, Indonesian, in 1994, and the M.Sc. degrees in Actuarial from the Bandung Institute of Technology (ITB), Bandung, Indonesian, in 2002. He is studying Ph.D. degrees in Informatics Engineering at University of Sriwijaya. In 1994, he joined, University of Sriwijaya, as a Lecturer. Since December 2006, he has been with the Department of Informatics Engineering, University of Sriwijaya, where he was an Assistant Professor, became an Associate Professor in 2011. Since 2012, he has been with the Department of Computer Engineering, University of Sriwijaya His current research interests include image processing, and computer vision. Erwin, S.Si., M.Si. is a member of IAENG and IEEE.

Saparudin was born in Pangkal Pinang, Indonesian, in 1969. He received the Bachelor degree in mathemathic education from the University of Sriwijaya, Indonesian, in 1993, and the M.Tech. degrees in informatics from the Bandung Institute of Technology (ITB), Bandung, Indonesian, in 2000 and Ph.D. degrees in computer science from the Malaysian University of Technology (UTM), Johor Bahru, Malaysian, in 2012. In 1995, he joined, University of Sriwijaya, as a Lecturer. Since December 2006, he has been with the Department of Informatics Engineering, University of Sriwijaya, where he was an Assistant Professor, became an Associate Professor in 2011, and a Professor in 2017. His current research interests include image processing, and computer vision. Drs. Saparudin, M.T., Ph.D. is a member of Institute of Advanced Engineering and Science (IAES) and IEEE.

Wulandari Saputri was born in Palembang, Indonesian, in 1996. She is student at Department of Computer Engineering, Faculty of Computer Science, University of Sriwijaya, Indonesia. In 2017, she joined the Laboratory of Image Processing, University of Sriwijaya, as Assistant Lecturer. Her current research interests include artificial intelligence, pattern recognition, computer vision, and image processing. 